\title{
Optical stochastic cooling of muons
}

\author{
A. Zholents and M. Zolotorev \\ Ernest Orlando Lawrence Berkeley National Laboratory, Berkeley, California 94720 \\ W. Wan \\ Fermi National Accelerator Laboratory, P.O. Box 500, Batavia, Illinois 60510
}

(Received 7 September 2000; published 5 March 2001)

\begin{abstract}
A draft scenario of optical stochastic cooling of muons is considered. Difficult places of this scenario are highlighted and constraints are explained. The parameters of a $2 \mathrm{TeV} \times 2 \mathrm{TeV}$ muon collider utilizing muons after optical stochastic cooling are presented.
\end{abstract}

DOI: 10.1103/PhysRevSTAB.4.031001

PACS numbers: 29.27.-a

\section{PREAMBLE}

This paper summarizes a feasibility study of optical stochastic cooling (OSC) of muons. We developed a draft scenario of OSC and considered what it would involve to implement it. In several places of this scenario we accepted a risky strategy because we could not otherwise find a plausible solution. This paper highlights these difficult places and explains our constraints.

In the first section of this paper we give a brief introduction to the OSC itself. In the next section, we present all formulas needed for a calculation of the damping time provided by the OSC, gain of the optical amplifier, optical energy, and power. Most of the formulas are given without a derivation, which can be found in Ref. [1]. A reader following the derivation of formulas in Ref. [1] will catch minor differences between the present and "old" formulas, which are due to the broader applicability of the present formulas.

The cooling scenario is considered in the third section. Here we explain the logistics of this scenario and highlight the places where we encountered difficulties that required the use of risky strategies. In the last section we consider the application of the cooling scenario developed in the preceding section to the $2 \mathrm{TeV} \times 2 \mathrm{TeV}$ muon collider.

\section{INTRODUCTION}

Recall that OSC obeys the same cooling principles as the microwave stochastic cooling proposed by van der Meer [2]. All modifications are associated with a transition into the range of optical frequencies [3]. The cooling system, referred to as a cooler, includes two undulators, an amplifier, and a bypass. The undulators serve as a pickup and a kicker, and the amplifier is an optical amplifier. By passing this system, the particle radiates an electromagnetic (EM) light wave in the upstream undulator. This wave is amplified in the optical amplifier while the particle goes through the bypass. Then, the particle meets the EM wave of its own amplified radiation in the downstream undulator. A subsequent interaction of the particle with the EM wave changes its energy. We define this process as an energy kick to the particle. There is also a nonzero lattice dispersion function in the undulator. It allows the coupling of the energy change to the transverse motion of the particle and produces what we define as a coordinate kick to the particle. ${ }^{1}$ The magnitude of the energy and coordinate kicks depends on the phase of the EM wave at the time when the particle enters the downstream undulator. This phase is defined by the particle energy and transverse coordinates in the pickup undulator and by the time-of-flight properties of the bypass lattice. One can adjust the delay time for the EM wave in the amplifier such that the equilibrium particle meets its EM wave at a phase when it can pass the undulator without an energy change. We will call this a zero phase. Then, time-of-flight properties of the bypass lattice can be used to regulate the phase shift from a zero phase, which is due to the particle coordinate offset and the particle energy offset. This gives control over the magnitude of the coordinate and energy kicks. This is how the undulator works as an energy and coordinate kicker. Cooling occurs as a result of the multiple beam passes through the cooler and an accumulation of a large number of correcting kicks. In the process of cooling, the coordinate and energy offsets of particles are decreased. Correspondingly, this cooling causes a decrease in the phase shifts and a reduction of the energy kicks associated with them. The latter is prevented by a continuous adjustment of the time-of-flight properties of the bypass lattice. The gain of the optical amplifier is also reduced following the reduction of the beam emittance and energy spread.

The method of stochastic cooling widely employs the concept of a bunch sample [2,4]. In the OSC, this is defined as a group of bunch particles that is capable of affecting each other by their radiation fields in the kicker undulator. The length of the sample depends on the bandwidth of the cooling system. Fast cooling requires short samples and, therefore, a wide bandwidth. It is also necessary that

\footnotetext{
${ }^{1}$ Typically, the dispersion function and the coordinate kick are in the horizontal plane.
} 
sample particles appear in the pickup undulator in the same order as in the kicker undulator with an accuracy of $\sim \lambda / 2 \pi$, where $\lambda$ is the period of the EM wave. However, this order must be deliberately mixed after the kicker undulator [4]. The randomization of the beam samples should occur between any two neighboring cooling systems in the ring. Thus, each cooler must be complemented by a mixer - a lattice designated to produce the fast randomization of the beam samples. For a more detailed description of the OSC we now refer the reader to Ref. [1].

\section{USEFUL FORMULAS}

In the case of OSC, the sample particles reside within the bunch slice of the length $M \lambda / 3$, where $\lambda$ is the central wavelength of the gain spectra of the optical amplifier and $M$ is the number of undulator periods. The shorter the sample the more sample slices in the bunch and, thus, the fewer particles residing in each sample. In stochastic cooling a small number of sample particles is a most important condition for a fast cooling [4]. For a given number of muons in the bunch $N$, the number of sample particles can be calculated as

$$
N_{s}=N \frac{M \lambda}{3 \ell_{b}},
$$

where $\ell_{b}$ is the bunch length. In principle, $N_{s}$ can be further reduced using so-called optical transverse sampling [1,5], but it turned out to be impractical in the case of muon cooling. We will come back to this point later.

The formulas for the inverse damping time of longitudinal and transverse oscillations are

$$
\begin{aligned}
\frac{1}{n_{\|}}=\frac{1}{e\left(N_{s}+n\right)} f_{\|}(x, r), & & \left|f_{\|}(x, r)\right| \leq 1, \\
\frac{1}{n_{\perp}}=\frac{1}{e\left(N_{s}+n\right)} f_{\perp}(x, r), & & \left|f_{\perp}(x, r)\right| \leq 1,
\end{aligned}
$$

where $e$ is the base of the natural logarithm, $n_{\|}, n_{\perp}$ stand for the number of passes through the cooling system needed for $1 / e$ reduction of the beam energy spread and emittance, $n \simeq 50$ is the optical amplifier noise factor that we will discuss later, and $f_{\|}, f_{\perp}$ are functions of the two parameters. The parameter $r=\sigma_{s} / \sigma_{b}$ is the ratio of the transverse synchrotron beam size $\sigma_{s}$ to the betatron beam size $\sigma_{b}$ in the pickup and kicker undulators. It shows the amount of the energy kick to be transformed to the coordinate kick. ${ }^{2}$ The parameter $x=\Delta \phi_{b} / \Delta \phi_{e}$ is the ratio of the two phase shifts discussed in the introduction: $\Delta \phi_{b}$ is the phase shift of the particle with zero energy offset and rms amplitude of betatron oscillations, and $\Delta \phi_{e}$ is the phase shift of the particle with the rms energy

\footnotetext{
${ }^{2}$ Equal dispersion and beta functions in the pickup and kicker undulators and the unit transfer matrix $I$ between undulators are assumed.
}

offset and zero amplitude of betatron oscillation. This parameter shows the origin of the energy kick, i.e., to what extent it is due to the coordinate or energy offset. The explicit expressions for $f_{\|}, f_{\perp}$ are as follows:

$$
\begin{aligned}
& f_{\|}(x, r)=\frac{2(1-r x)-\frac{1}{1+r^{2}}}{\left(1+r^{2}\right)\left[x^{2}+(r x-1)^{2}\right]}, \\
& f_{\perp}(x, r)=\frac{2 r x-\frac{r^{2}}{1+r^{2}}}{\left(1+r^{2}\right)\left[x^{2}+(r x-1)^{2}\right]} .
\end{aligned}
$$

Figure 1 shows $f_{\|}$and $f_{\perp}$ for four values of $r$ and $-2 \leq$ $x \leq 2$. Note that $f_{\|}=f_{\perp}=1 / 2$ at $x=0.5$ and $r=1$. Then Eq. (2) reduces to Eq. (10b) of Ref. [1].

To cool with the rates of Eq. (2) an optical amplifier is needed with an amplitude signal gain $g$ :

$$
\begin{aligned}
g & \simeq \frac{1}{4 \pi \sqrt{e}\left(N_{s}+n\right)} \frac{\left(2+K^{2}\right)}{K^{2}} \frac{\gamma \lambda \sigma_{e}}{r_{\mu}} f_{g}(x, r), \\
f_{g}(x, r) & =\frac{1}{\left(1+r^{2}\right) \sqrt{x^{2}+(r x-1)^{2}}}, \\
0 & \leq f_{g}(x, r) \leq 1,
\end{aligned}
$$

where $\gamma$ is the Lorentz factor, $\sigma_{e}$ is the relative beam energy spread, $K=\mathbf{e} B_{0} \lambda_{u} / 2 \pi m c^{2}$ is the undulator parameter, $B_{0}$ is the undulator peak magnetic field, $m$ is the muon mass, $\lambda_{u}$ is the undulator period, $\mathbf{e}$ is the muon charge, and $c$ is the speed of light.

The gain-narrowed bandwidth of the amplifier is defined in Ref. [6] as

$$
\frac{\Delta f}{f} \simeq \frac{2 \Gamma}{\ln (g)},
$$

where $\Gamma \simeq 0.4$ is the relative bandwidth of the $\mathrm{Ti}_{\mathrm{Al}} \mathrm{Al}_{2} \mathrm{O}_{3}$ crystal. This is the gain medium for the optical amplifier considered at the time of this writing $(\lambda \simeq 800 \mathrm{~nm})$. Assuming that the bandwidth of the particle radiation in the pickup and kicker undulators matches the amplifier bandwidth, we define the number of undulator periods as
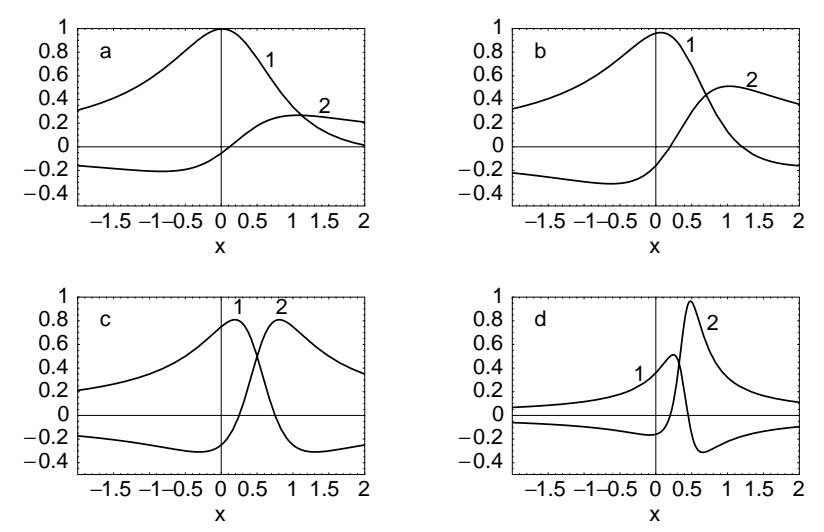

FIG. 1. The functions $f_{\|}$(curve 1) and $f_{\perp}$ (curve 2) versus $x$ : (a) $r=0.25$, (b) $r=0.5$, (c) $r=1$, and (d) $r=2$. 


$$
\frac{1}{M} \simeq \frac{\Delta f}{f} .
$$

A number of signal photons at the amplifier front end produced by one bunch sample is equal to

$$
N_{\mathrm{ph}} \simeq 4 \alpha \frac{K^{2}}{\left(2+K^{2}\right)} N_{s},
$$

where $\alpha=1 / 137$ is the fine structure constant.

The dominant source of the amplifier noise is spontaneous emission in the gain medium. It gives one photon per mode at the amplifier front end [6] — a theoretical minimum reached in practice. It is convenient to consider this photon as if it is produced in the pickup undulator by some number $n$ of fictitious sample particles. Therefore, in order to account for the effect of the amplifier noise on damping in Eqs. (2a) and (2b) we simply added $n$ to a real number of sample particles. Using Eq. (7), with $N_{\mathrm{ph}}=1$ and $N_{s}=n$, we find

$$
n=\frac{1}{4 \alpha} \frac{\left(2+K^{2}\right)}{K^{2}} .
$$

The upper limit for $K$ is imposed by the peak magnetic field in the undulator. We show later that it is already $\sim 10 \mathrm{~T}$ at $K=2$. Therefore, using $K=2$ we find $n \simeq 50$.

Finally, the light energy $\delta E$ provided by all optical amplifiers during the cooling process can be estimated as a product of the total number of photons at the amplifier front end including noise $4 \alpha \frac{K^{2}}{\left(2+K^{2}\right)}\left(N_{s}+n\right) \frac{N}{N_{s}}$, where $N / N_{s}$ is the number of the beam samples, photon energy $\hbar \omega$, square of the amplitude gain $g$ at the beginning of cooling, and a number of beam passes through the cooler during one damping time. Then, using Eqs. (1), (2b), and (4), $x=0.5$ and $r=1$, we obtain

$$
\delta E \simeq \frac{\epsilon_{\|} \sigma_{e} E_{b}}{M r_{\mu}},
$$

where $\epsilon_{\|}$is the normalized longitudinal emittance, $E_{b}$ is the muon beam energy, and $r_{\mu}$ is the classical radius of muon. Thus, the average power of all optical amplifiers is $\delta E f_{0}$, where $f_{0}$ is the repetition rate of cooling cycles. More information about the optical amplifier for OSC can be found in Ref. [7].

\section{COOLING SCENARIO}

\section{A. Beam energy}

The consideration of a cooling scenario begins from a choice of the beam energy for cooling. It is constrained by the so-called resonance condition:

$$
\lambda=\frac{\lambda_{u}}{2 \gamma^{2}}\left(1+K^{2} / 2\right) .
$$

This is simply a requirement that the undulator radiation of muons be matched to the spectral gain function of the amplifier. For a given $K$ and $\lambda$ the condition of Eq. (10)
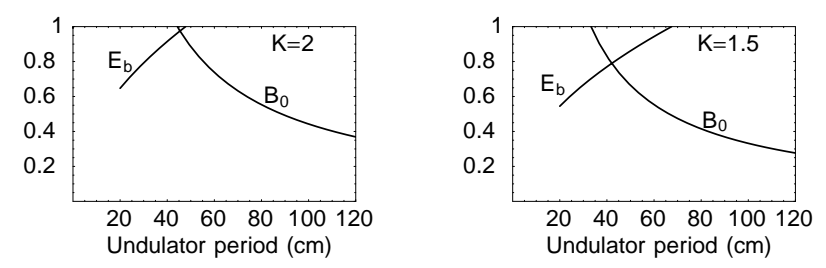

FIG. 2. Beam energy $E_{b}$ in units of $100 \mathrm{GeV}$ and peak undulator field $B_{0}$ in units of $10 \mathrm{~T}$ as a function of the undulator period for two values of undulator parameter $K$.

can be satisfied with different sets of $E_{b}, B_{0}$, and $\lambda_{u}$. However, if we allow only energies below $100 \mathrm{GeV}$ and a magnetic field below $10 \mathrm{~T}$, then the choice is limited. Figure 2 shows $E_{b}$ (in units of $100 \mathrm{GeV}$ ) and $B_{0}$ (in units of $10 \mathrm{~T}$ ) as functions of $\lambda_{u}$ for two values of $K$ and $\lambda=800 \mathrm{~nm}$. At $K=2$ both functions can be crossed by the same vertical line [indicating a solution of Eq. (10)] only within a tiny range of $\lambda_{u}$ near $\lambda_{u} \simeq 50 \mathrm{~cm}$. More choices appear at $K=1.5$. However, going from $K=2$ to $K=1.5$ leads to $\sim 20 \%$ decrease in $N_{\mathrm{ph}}$ and $\sim 20 \%$ increase in $n$ [see Eqs. (7) and (8)]. At the time of this writing, we decided to stay with $E_{b}=100 \mathrm{GeV}, B_{0}=10 \mathrm{~T}$, and $K=2$.

\section{B. Damping time}

Obviously, to cool muons we want fast damping. Here we investigate what is possible with the OSC. One cooling system in OSC allows us to cool the longitudinal emittance and either the horizontal or vertical emittance [1]. To cool both transverse emittances we need to alternate cooling in the horizontal and vertical planes. Therefore, we want to cool each transverse emittance at least twice as fast as the longitudinal emittance (which is 2.5 times faster in the practical example considered at the end of the paper). This leads us to a parameter choice of $x \simeq 0.61$ and $r=$ 1 , resulting in $f_{\|}=0.27$ and $f_{\perp}=0.69$ [see Eq. (3)]. Then, according to Eq. (3) we have $n_{\|} \simeq 10\left(N_{s}+n\right)$ and $n_{\perp} \simeq 4\left(N_{s}+n\right)$. Thus, even with $N_{s}=n / 2=25$ (signal-to-noise ratio of $1 / 2, n=50$ as above), we get $n_{\|} \simeq 750$ and $n_{\perp} \simeq 300$. One interesting observation is that Eq. (7) with $N_{s}=25$ gives us $N_{\mathrm{ph}}=1 / 2$. It means that, on average, sample muons radiate an actual photon once per every two passes through the cooling system. Therefore, a $1 / e$ reduction of the transverse emittance will take an average of $\sim 150$ correcting kicks for each particle. This is still a reasonably large number, so we may not be concerned much with statistical fluctuations of correcting kicks. However, going further down to, say, $N_{s}=12$ will reduce the number of correcting kicks to $\sim 75$ while giving us only a little faster cooling time of $n_{\perp} \simeq 250$. This is not a big bonus considering the penalty of having $\sqrt{2}$ times larger statistical fluctuations. Therefore we have decided to stay with $N_{s}=25$.

We show later that in order to explore most benefits from OSC we need to reduce the 6D beam emittance by $\sim 4 \times 10^{10}$ times. This translates to 8.8 damping times 
of cooling for each transverse direction and 6.9 damping times of cooling for the longitudinal direction. Thus, we would need $6.9 \times 750 \simeq 5200$ passes through the cooler. A preliminary design of the cooler lattice has led us to the conclusion that the lower limit for its length is $\sim 80 \mathrm{~m}$. A similar design for a mixer gave us $\sim 30 \mathrm{~m}$. Thus, with a minimal length $110 \mathrm{~m}$ for the cooling system and 5200 passes we get a minimum $2 \mathrm{~ms}$ of cooling time. This is equal to approximately one muon decay time at a $100 \mathrm{GeV}$ muon energy.

\section{Optical amplifier}

Equations (4) and (9) illustrate the fact that we benefit in both cases from a small energy spread. Using $\lambda=$ $800 \mathrm{~nm}, \sigma_{e}=2 \times 10^{-6}$ (the smallest $\sigma_{e}$ that we were prepared to accept at the time of this writing), and other parameters as above defined, we calculate $f_{g}=0.7$ and $g \simeq 3.8 \times 10^{4}$. Then, using Eqs. (5) and (6), we find that $M \simeq 14$.

For a bunch length we assume $\sigma_{z}=100 \mathrm{~m}$. As we see later, this is still a manageable length. Using this $\sigma_{z}$ and the above-defined $\sigma_{e}$ we calculate for normalized longitudinal emittance $\epsilon_{\|} \simeq 20 \mathrm{~cm}$. This longitudinal emittance is larger by an approximate factor of 5 than what is presently considered in the "conventional" design of muon colliders [8]. It is assumed that the required longitudinal emittance can be obtained by means of a correspondent reduction of the transverse emittance. The practical realization of this emittance exchange is not considered in this study and is yet to be defined

Now we are able to calculate the light energy $\delta E$ using Eq. (9). It gives us $\delta E=10 \mathrm{~J}$ or $\sim 1 \mathrm{~J}$ of the light energy per amplifier during the cooling assuming ten optical amplifiers. It translates into $200 \mathrm{~W}$ of the average output power at a repetition rate of $f_{0}=200 \mathrm{~Hz}$. The amplifier considered in Ref. [7] is much more modest, giving approximately $2 \mathrm{~W}$ of average power. However, it is based on a rather standard design that follows a design of commercially available lasers. More sophisticated lasers using liquid-nitrogen cooled Ti:sapphire crystal have been built and tested at $350 \mathrm{~W}$ of average power [9]. It seems possible to use a similar advance approach to build a high average power amplifier. One can also notice that dealing with a long light pulse coming from a muon bunch is rather convenient for optical amplification. It may allow an adjustable gain to have a continuous compensation of a depletion of the excited states in the amplifier medium during the bunch passage.

\section{Cooling scheme}

Now we have all of the necessary ingredients to characterize the muon beam entering the OSC chain. If we take the $6 \mathrm{D}$ normalized beam emittance of $10^{-4} \mathrm{~cm}^{3}$ obtained in the ionization cooling [8] and use the above-defined longitudinal emittance of $20 \mathrm{~cm}$, we get $\epsilon_{\perp} \simeq 2 \times 10^{-3} \mathrm{~cm}$ for the normalized transverse emittance. Next, using $\ell_{b}=$ $2.5 \sigma_{z}$, we find from Eq. (1) that $N \simeq 2 \times 10^{9}$. This is the number of muons that we would be able to cool with the above-defined rate.

We assume that a primary muon beam will be accelerated to $100 \mathrm{GeV}$ with a relatively short bunch length of $\sigma_{z}=20 \mathrm{~cm}$ and an energy spread of $\sigma_{e}=10^{-3}$. Then, it has to be stretched to $\sigma_{z} \simeq 10^{4} \mathrm{~cm}$ with the correspondent reduction of the energy spread. To do this we use a stretcher (shown in Fig. 3) as the first component of the scheme. This is a $100 \mathrm{GeV}$ storage ring with a $\sim 300 \mathrm{~m}$ circumference. A distinctive feature of this ring is a large momentum compaction factor of $\alpha=0.33$. (A preliminary design of the ring lattice had proved that this $\alpha$ is feasible.) With this $\alpha$ the beam will be stretched in $\sim 1000$ turns $(\sim 1 \mathrm{~ms}$ time $)$, which is approximately equal to one-half of the muon decay time at $100 \mathrm{GeV}$. To account for the loss of muons in the stretcher we would need to begin stretching with $N \simeq 3 \times 10^{9}$ muons. The muon beam leaves the stretcher with the correlated head-to-tail energy increment of $250 \mathrm{MeV}$ measured at a $2.5 \sigma$ level.

The next component of the cooling scheme is an induction linac. Its function is to cancel the energy variation along the bunch. Therefore, it has a sawtooth pulse of $\sim 1 \mu$ s duration to accommodate a $\sim 250 \mathrm{~m}$ long muon bunch. The amplitude of the acceleration along the bunch ramps linearly, so the head-to-tail variation of the acceleration gain at a $\pm 1.25 \sigma$ level consists of $250 \mathrm{MeV}$. The linearity of the ramp is expected to be better than $10^{-3}$ to preserve the small energy spread of muons.

An actual damping takes place in three damping rings stacked one on top of the other. We need three rings to accomplish a deep detuning of the time-of-flight parameters of the ring lattice in a short period of time. The muon beam stays in each ring for approximately three transverse damping times. The rings are very similar and share optical amplifiers while the muon beam is moving from one ring to another. Each ring has ten identical cooling

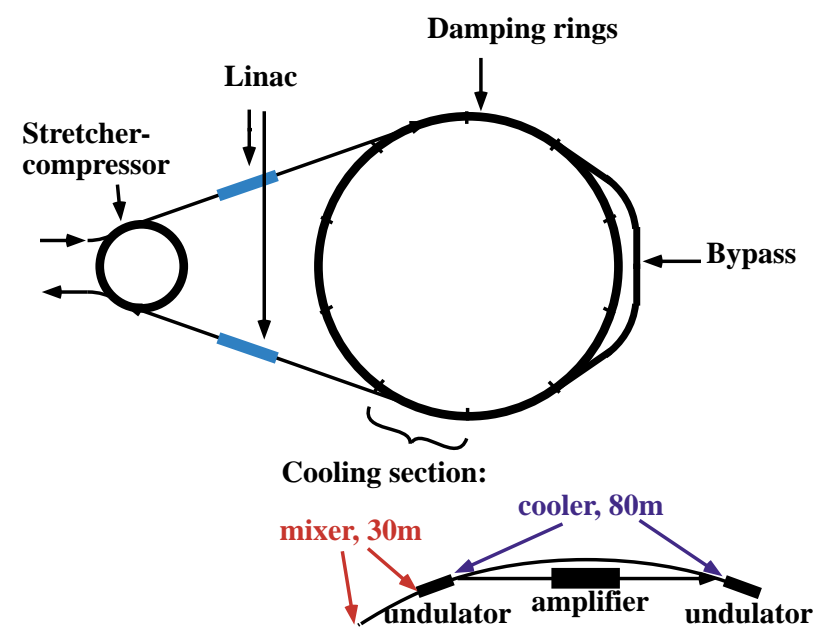

FIG. 3. (Color) The cooling scheme. 
sections, each consisting of a cooler and a mixer that build up to a circumference of $1100 \mathrm{~m}$.

The lattice of the cooling section has certain specifics. Recall that earlier we used $r=\sigma_{s} / \sigma_{b}=1$. Now, using the actual energy spread and emittance of the muon beam, we find $D_{x} \simeq 100 \mathrm{~m}$ for a horizontal dispersion function in the pickup and kicker undulators, assuming $\beta_{x}=2 \mathrm{~m}$ for a horizontal beta function. This is a huge disproportion in the lattice functions, and it takes a special lattice of at least $\sim 100 \mathrm{~m}$ long to create it. Therefore, we have decided to create this disproportion outside of the ring in the beam line connecting the induction linac and the first damping ring keeping the ring itself unchanged. However, this is a poor man's solution since it requires operation of the ring at an integer resonance. A plausible compromise has been to add a bypass to the ring. The idea is to turn off the muon beam into the bypass every $\sim 30$ turns for a detuning from the integer resonance. The bypass lattice includes dispersion suppression lattices on both sides and a dispersion free central part. The central part serves two purposes. The first purpose is the detuning from the integer resonance. Further studies are needed to find out a necessary amount of a tune shift to keep from growing the amplitudes of particle betatron oscillations. The second purpose is the rotation of the horizontal and vertical betatron oscillations. Recall that we have been talking about alternating the cooling in the horizontal and vertical planes. Here is how it works. The dispersion function in the ring coolers is in the horizontal plane. Thus, cooling in the ring is shared between the energy cooling and cooling of the horizontal betatron oscillations. However, while in the bypass, the horizontal mode of particle motion is turned into the vertical mode of particle motion and vice versa. Thus producing the alternating cooling of the horizontal and vertical emittances at every $\sim 30$ turns.

One of the reasons we have had to abandon the idea of transverse samples for muon cooling is the large $D_{x}$ requirement. We would need at least 10 to 100 times larger $D_{x}$ to achieve $r \simeq 1$ with transverse samples. This seems impractical.

In the Introduction we emphasized the necessity of adjusting the time-of-flight characteristics of the cooler lattice. We studied this issue during the lattice design and found that with $\leq 15 \%$ trimming of the strength of the quadrupole lenses we could cover the range of adjustments of time-of-flight characteristics of the lattice sufficient to follow beam cooling during $\sim 3$ damping times. A larger than $15 \%$ trimming seems difficult because it creates large perturbations in the lattice. Even a $15 \%$ trimming range may turn out to be too large because the adjustments should be done in a time period of $\sim 700 \mu$ s. As a result we have decided to use three damping rings instead of one, since we need to cool for approximately nine damping times. Moreover, all adjustments of time-of-flight characteristics should be performed to the level of accuracy $\sim \lambda / 2 \pi$. It seems feasible based on a preliminary study reported in
Ref. [10], but more work is needed to demonstrate it for a dynamically changed lattice.

Cooling ends with the following result: $\epsilon_{\perp} \simeq 3 \times$ $10^{-7} \mathrm{~cm}, \epsilon_{\|} \simeq 2 \times 10^{-2} \mathrm{~cm}$, and $N \simeq 7 \times 10^{8}$.

To compress the muon bunch after cooling, we plan to send it to the induction linac for the head-to-tail energy modulation and after that to proceed with the actual compression in the ring with the large $\alpha$. We are going to use the same ring for compression as for stretching. The entire process will take approximately $1 \mathrm{~ms}$, and it will bring down further the number of muons due to a muon decay to $4.5 \times 10^{8}$.

In Table I we combine all beam parameters and parameters of the cooling scheme defined above.

TABLE I. List of the beam parameters and parameters of the cooling scheme.

\begin{tabular}{|c|c|c|}
\hline & Units & Value \\
\hline Beam energy & $\mathrm{GeV}$ & 100 \\
\hline Repetition rate & $\mathrm{Hz}$ & 200 \\
\hline \multicolumn{3}{|l|}{ Input beam characteristics } \\
\hline Number of muons & & $3 \times 10^{9}$ \\
\hline Transverse emittance & $\mathrm{cm} \mathrm{rad}$ & $2 \times 10^{-3}$ \\
\hline Longitudinal emittance $\left(\gamma \sigma_{z} \sigma_{e}\right)$ & $\mathrm{cm}$ & 20 \\
\hline Beam energy spread $\left(\sigma_{e}\right)$ & & $1 \times 10^{-3}$ \\
\hline Bunch length $\left(\sigma_{z}\right)$ & $\mathrm{cm}$ & 20 \\
\hline \multicolumn{3}{|l|}{ Stretcher-compressor } \\
\hline Circumference & $\mathrm{m}$ & 300 \\
\hline Momentum compaction & & 0.33 \\
\hline \multicolumn{3}{|l|}{ Induction linac } \\
\hline Pulse duration & $\mu \mathrm{s}$ & 1 \\
\hline Energy gain & $\mathrm{MeV}$ & \pm 125 \\
\hline \multicolumn{3}{|l|}{ Damping rings } \\
\hline Circumference & $\mathrm{m}$ & 1100 \\
\hline Number of rings & & 3 \\
\hline Number of injected muons & & $2 \times 10^{9}$ \\
\hline Beam energy spread $\left(\sigma_{e}\right)$ & & $2 \times 10^{-6}$ \\
\hline Bunch length $\left(\sigma_{z}\right)$ & $\mathrm{m}$ & 100 \\
\hline Number of sample particles & & 25 \\
\hline Longitudinal damping time & turns & 75 \\
\hline Transverse damping time & turns & 30 \\
\hline Undulator period & $\mathrm{cm}$ & 50 \\
\hline Peak undulator field & $\mathrm{T}$ & 10 \\
\hline Number of periods & & 14 \\
\hline Dispersion function & $\mathrm{m}$ & 100 \\
\hline Beta function & $\mathrm{m}$ & 2 \\
\hline \multicolumn{3}{|l|}{ Optical amplifier } \\
\hline Number of amplifiers & & 10 \\
\hline Amplified light energy & $\mathrm{J}$ & 1 \\
\hline Average output power & $\mathrm{W}$ & 200 \\
\hline Amplitude gain & & $3.8 \times 10^{4}$ \\
\hline Wavelength & $\mathrm{nm}$ & 800 \\
\hline \multicolumn{3}{|l|}{ Output beam characteristics } \\
\hline Number of muons & & $4.5 \times 10^{8}$ \\
\hline Transverse emittance & $\mathrm{cm} \mathrm{rad}$ & $3 \times 10^{-7}$ \\
\hline Longitudinal emittance & $\mathrm{cm}$ & $2 \times 10^{-2}$ \\
\hline Cooling time ${ }^{\mathrm{a}}$ & $\mathrm{ms}$ & 4 \\
\hline
\end{tabular}

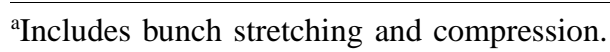


TABLE II. List of the parameters for a $2 \mathrm{TeV} \times 2 \mathrm{TeV}$ muon collider.

\begin{tabular}{lccc}
\hline \hline & Units & This study & Ref. [8] \\
\hline Beam energy & $\mathrm{TeV}$ & 2 & 2 \\
Circumference & $\mathrm{km}$ & 8.08 & 8.08 \\
Number of muons & & $4.5 \times 10^{8}$ & $2 \times 10^{12}$ \\
$\begin{array}{l}\text { Number of bunches for } \\
\text { each charge }\end{array}$ & 2 & 2 \\
Beta function at the IP & $\mu \mathrm{m}$ & 10 & 3000 \\
Bunch length & $\mu \mathrm{m}$ & 10 & 3000 \\
Peak current & $\mathrm{kA}$ & 2 & 32 \\
Transverse beam size & $\mu \mathrm{m}$ & $1.3 \times 10^{-3}$ & 3 \\
$\quad$ at the IP & & $1.3 \times 10^{-4}$ & $1 \times 10^{-3}$ \\
Beam divergence at the IP & & $1 \times 10^{-3}$ & $7 \times 10^{-4}$ \\
Beam energy spread & & 0.15 & 0.045 \\
Beam-beam parameter & $\mathrm{Hz}$ & 200 & 15 \\
Repetition rate & $\mathrm{cm}^{-2} \mathrm{~s}^{-1}$ & $1 \times 10^{35}$ & $1 \times 10^{35}$ \\
Luminosity & &
\end{tabular}

${ }^{\mathrm{a}}$ This is the rate for a muon production line.

\section{APPLICATION TO THE COLLIDER}

Here we consider the $2 \mathrm{TeV} \times 2 \mathrm{TeV}$ muon collider. A list of the most essential parameters is included in Table II. For the convenience of the reader we also include in Table II the same list of parameters of the muon collider obtained in a conventional design (CD) (Ref. [8]).

Here are a few comments regarding a comparison of the parameters. The bunch length is unusually short, but the peak current is actually much smaller than that found in the CD. Coherent synchrotron radiation at $\sigma_{z} \sim 10 \mu \mathrm{m}$ is practically shielded by the vacuum chamber and produces a negligible energy loss. The beta function is extremely small, but the beam divergence at the interaction point (IP) is also small, smaller than in the CD. Therefore, it should not be anymore difficult to obtain this beta function than the CD's beta function. The beam-beam parameter seems too large, but according to the study [11] performed with the CD's beam parameters, it is marginally acceptable for $\sim 350$ orbit turns $(\sim 10 \mathrm{~ms})-$ a time between injection cycles into the same bucket. The number of muons in the collider is down by a factor of $4.4 \times 10^{3}$. The number of muons in the production line that includes a proton driver and ionization cooling is also down by a factor of $\sim 50$.

\section{SUMMARY}

This study provides a realistic assessment of the OSC of muons. We assume that the OSC is implemented after the ionization cooling of muons and acceleration to $100 \mathrm{GeV}$ - the preferable energy for OSC. The OSC takes approximately one muon decay time. One extra decay time is spent for a phase space rotation of the muon beam before and after cooling. The $6 \mathrm{D}$ emittance shrinks more than a factor of $10^{10}$ during cooling. This allows us to obtain the same design luminosity as in the conventional design of a muon collider using $4.4 \times 10^{3}$ fewer muons per beam. For this reason, the proton driver can supply a beam of protons of approximately 50 times less pulse intensity than what is in the conventional design. This already accounts for extra losses of muons occurring after the ionization cooling.

A significant reduction in the number of muons in a collider ring will have the result of a dramatic reduction of fluxes of neutrinos and electrons produced in the muon decay. There should also be an improvement in the background in the detector. These three factors are the main advantage to using the OSC for a muon collider.

On the other hand, the OSC significantly increases the complexity of the muon collider facility. For each type of muon we use three damping rings, one stretchercompressor ring, and two induction linacs. Some of these systems could possibly be shared between positively and negatively charged muons, but only at the expense of increased complexity of the operation. All damping rings are of the same circumference and could be placed in the same tunnel. In this way they can share ten optical amplifiers. The optical amplifier has rather advanced characteristics, that, to the best of our knowledge, exceed the parameters of optical amplifiers that can be routinely made at the time of this writing.

Overall, we conclude that OSC of muon is difficult and expensive. Further studies are needed to decide whether the benefit of the additional cooling outweighs the great complexity and considerable cost associated with its implementation.

\section{ACKNOWLEDGMENTS}

We are grateful to S. Chattopadhyay, R. Palmer, A. Sessler, and J. Wurtele for numerous useful discussions and their interest in this work. This work was supported 
by the U.S. Department of Energy under Contracts No. DE-AC03-76SF00098 and No. DE-AC02-CH03000.

[1] M. Zolotorev and A. Zholents, Phys. Rev. E 50, 3087 (1994).

[2] S. van der Meer, CERN Report No. CERN/ISR PO/72-31, 1972 (unpublished).

[3] A. Mikhalichenko and M. Zolotorev, Phys. Rev. Lett. 71, 4146 (1993).

[4] D. Möhl, CERN Report No. 87-03, 1987, p. 453.

[5] K.-J. Kim and A. Zege, in Proceedings of the Particle Accelerator Conference, Dallas, Texas, 1995 (IEEE, Piscataway, NJ, 1996), pp. 2786-2788.
[6] A.E. Siegman, Lasers (University Science Books, Mill Valley, CA, 1986).

[7] A. Zholents and M. Zolotorev, in Proceedings of the Particle Accelerator Conference, Vancouver, Canada, 1997 (IEEE, Piscataway, NJ, 1998), pp. 1804-1806.

[8] "Muon Muon Collider: Feasibility Study," BNL-52503, Fermi Lab-conf.-96/092, LBNL-38946, 1996.

[9] P. A. Schultz and S. R. Henion, IEEE J. Quantum Electron. 27, 1039 (1991).

[10] A. Zholents, M. Zolotorev, and W. Wan, in Proceedings of the Particle Accelerator Conference, Vancouver, Canada, 1997 (Ref. [7]), pp. 1801-1803.

[11] M. Furman (private communication). 